\title{
Evolução das Características Ecoggráficas da Placenta, da Posição e da Apresentação Fetal em Gestações Normais.
}

\author{
Evolution of Ultrasound Characteristics of Placenta and Fetal Position and \\ Presentation in Normal Pregnancies.
}

Maria Regina Machado Perrotti, José Guilherme Cecatti, Milton Bricola Filho Kleber Cursino de Andrade

\section{RESUM0}

Objetivo: avaliar as características ecográficas da gestação normal, segundo o grau, a localização e a espessura placentária, a apresentação e a posição fetal ao longo da segunda metade da gestação.

Métodos: estudo descritivo, incluindo no mínimo 120 medidas em cada idade gestacional, de 2.868 gestantes normais da cidade de Campinas, por meio de exame ultra-sonográfico de rotina, considerando-se os critérios de Grannum, Berkowitz, Hobbins (1979) para a classificação do grau placentário e a medida da espessura placentária no local da inserção do cordão umbilical.

Resultados: a placenta grau O foi mais comum até 31 semanas, o grau I apresentou maior freqüência após a $32^{a}$ semana e o grau II não foi observado antes da $32^{\underline{a}}$ semana. A placenta grau III foi mais freqüente a partir da $36^{a}$ semana. A espessura placentária aumentou significativamente com a gestação. As localizações mais freqüentes foram a anterior e a posterior. A apresentação cefálica foi a mais freqüente em todas as idades gestacionais, com apenas $1 \%$ de apresentações pélvicas ao termo. A posição fetal mais freqüente foi a de dorso lateral esquerda, seguida da de dorso lateral direita.

Conclusões: os fatores estudados tiveram distribuição similar à esperada para populações normais e podem servir como um padrão para a população brasileira.

PALAVRAS-CHAVE: Placenta. Gravidez normal. Ultra-sonografia.

\section{Introdução}

O bem-estar fetal e o bom resultado perinatal são os objetivos da assistência obstétrica. No entanto, é necessário conhecer e interpretar as reações do feto para que se possa avaliar suas condições intra-útero. Com a realização do exame ultra-sonográfico obstétrico tornou-se mais fácil antecipar o diagnóstico de alguma forma de comprometimento fetal ou comprovar seu bemestar $^{1}$. Dentre estas informações estão aquelas referentes à placenta, posição e apresentação fetal.

Serviço de Ecografia do Centro de Atenção Integral à Saúde da Mulher e "Medical Center - Diagnose - Serviço de Ecografia e Monitorização Fetal”, Campinas, SP

Correspondência:

José Guilherme Cecatti

Caixa Postal 6030

13081-970 - Campinas - SP

Fax: (19) 788-9304

Este estudo foi financiado pela FAPESP (processo 97/05382-4)
O exame ultra-sonográfico da placenta é de extrema importância e os parâmetros empregados na investigação são sua textura, grau de maturidade, localização e espessura. Em relação à textura da placenta, existe uma associação direta entre o grau de calcificação com o de maturidade pulmonar fetal, já conhecida há cerca de duas décadas ${ }^{2}$.

O grau de maturidade pode ser obtido pela intensidade, quantidade e extensão de calcificação da placenta, avaliado pelo exame ultra-sonográfico e expresso como um escore que varia de 0 a III. O grau 0 designa uma placenta homogênea, sem calcificação; o grau I, presença de pequenas calcificações intraplacentárias; o grau II, de calcificações na placa basal; e no grau III observase compartimentação da placenta pela presença de calcificação da placa basal à coriônica ${ }^{2}$.

A localização placentária corresponde à posição da placenta na cavidade uterina. Pode ser 
determinada pelo exame ultra-sonográfico que se apresenta como método não-invasivo, com boa acurácia para esta finalidade ${ }^{3}$. Pode ser classificada como: prévia, se qualquer parte da placenta está implantada sobre o segmento inferior do útero ${ }^{4}$; anterior, quando inserida na parede anterior uterina; posterior, quando inserida na parede posterior do útero; lateral, localizada na região lateral direita ou esquerda do útero; e fúndica, quando no fundo uterino.

Conhecer a localização da placenta é importante, principalmente quanto ao fato de ser prévia ou não, pela atenção que demanda em termos de cuidados pré-natais e inclusive quanto à via de parto. A ultra-sonografia pode ainda determinar as variedades de placenta prévia, desde lateral, marginal, centro-total ou centro-parcial ${ }^{3}$. O conhecimento destas variedades é também importante para a condução clínica destes casos, visto que geralmente estão associados a um maior risco materno e perinatal.

Tem-se dado importância também à espessura placentária pela sua relação com a presença de infecções materno-fetais e com a idade gestacional ${ }^{5}$. A espessura placentária é obtida por meio da medida a partir da placa basal até a placa corial, no ponto da inserção do cordão umbilical.

A posição fetal é a relação do dorso fetal com o lado direito ou esquerdo materno, podendo apresentar as alternativas: mediano anterior, mediano posterior, lateral direito ou lateral esquerdo. Já a apresentação é dada pelo pólo fetal que se relaciona com a área do estreito superior da bacia materna, podendo ser cefálica, pélvica ou córmica. Na maior parte dos casos isto pode ser determinado pela palpação do abdomen da gestante. Porém, em casos de dificuldade, o exame ultra-sonográfico pode ser de grande ajuda para a condução do parto e escolha de sua via.

Embora estes parâmetros placentários e fetais fornecidos pela ultra-sonografia estejam já incorporados à prática obstétrica, não existe de fato um verdadeiro conhecimento sobre sua distribuição durante as diferentes idades gestacionais em gestantes brasileiras normais. Dessa forma, a proposição deste estudo é avaliar a distribuição destas características numa grande população de gestantes normais, para estabelecer um padrão de normalidade e poder aplicar este conhecimento à clínica.

\section{Pacientes e Métodos}

O estudo realizado foi descritivo. Foram avaliadas, no Serviço de Ecografia do Centro de
Atenção Integral à Saúde da Mulher, da Universidade Estadual de Campinas e no "Medical Center - Diagnose - Serviço de Ecografia e Monitorização Fetal", ambos de Campinas, SP, no mínimo 120 gestantes para cada semana de idade gestacional, a partir da $20^{a}$ até a $42^{\text {a }}$ semana, totalizando 2.868 medidas independentes. Incluíram-se no estudo exames realizados no período de agosto de 1997 até abril de 1998.

Foi incluídos apenas os casos com data da última menstruação conhecida com certeza ou, caso esta primeira condição não fosse cumprida, com idade gestacional estimada por exame ultra-sonográfico pélvico precoce, realizado antes de 24 semanas; sem uso de anticoncepcionais orais nos últimos três meses ou injetáveis nos últimos seis meses anteriores a esta gestação, para que a idade gestacional fosse considerada confiável; e com idade gestacional de 20 a 42 semanas.

Foram excluídos da amostra os casos considerados como gestação não-normal, com hipertensão arterial crônica, hipertensão gestacional, diabetes mellitus, macrossomia fetal, rotura de membranas, senescência placentária precoce (segundo classificação de Grannum, Berkowitz, Hobbins ${ }^{2}$ ), gestação múltipla, retardo de crescimento intra-uterino, anomalia congênita fetal, óbito fetal, isoimunização fetal, outras situações tais como doenças metabólicas, nefropatias, cardiopatias, hiper e hipotiroidismo materno.

Para a realização dos exames ultrasonográficos propostos, foram utilizados os aparelhos Toshiba SSA-140® e Acuson XP4A ${ }^{\circledR}$, usando-se um transdutor convexo de $3,75 \mathrm{mHz}$ em ambos, normalmente adotado como padrão para exames ultra-sonográficos obstétricos. Todos os exames foram realizados pelo mesmo examinador.

Para se estudar a distribuição das características da atual gestação em relação à idade gestacional, inicialmente compararam-se descritivamente suas freqüências, médias e desvios-padrão para cada categoria de idade gestacional. A diferença entre elas foi avaliada por análise de variância para o caso de espessura da placenta, além da construção de uma curva de variação normal cujos extremos foram determinados por dois desvios-padrão acima e abaixo da média. Para o desenvolvimento destes procedimentos estatísticos utilizou-se o programa EPI INFO e o pacote "Statistical Package for Social Sciences” (SPSS/PC+).

Como o exame ultra-sonográfico durante a gestação não oferece riscos, quando realizado com as doses de ondas sonoras recomendadas, tanto para a mãe quanto para o feto, e em nada o estudo 
modificou este procedimento, não foi necessário o termo de consentimento informado da gestante para a coleta dos dados referentes ao exame. Respeitaram-se os termos da Declaração de Helsinki (1990). O estudo foi previamente aprovado pela Comissão de Ética em Pesquisa da instituição.

\section{Resultados}

Dentre as localizações da placenta, a mais freqüente foi a anterior, com $45,5 \%$, seguida da posterior com $42,9 \%$. As placentas prévias centro-totais corresponderam a $0,4 \%$ do total, as centro-parciais a $0,1 \%$ e as marginais a $0,2 \%$. A Tabela 1 mostra a freqüência das principais localizações placentárias.

Tabela 1 - Distribuição percentual da localização das placentas, segundo a idade gestacional.

\begin{tabular}{|c|c|c|c|c|c|c|}
\hline \multirow{2}{*}{$\begin{array}{l}\text { Idade Gestacional } \\
\qquad \text { (sem) }\end{array}$} & \multicolumn{5}{|c|}{ Localização placentária } & \multirow[b]{2}{*}{$\mathrm{n}$} \\
\hline & Anterior & Posterior & Lateral & Fúndica & Prévias & \\
\hline 20 & 46,0 & 43,5 & 2,4 & 6,5 & 1,6 & 124 \\
\hline 21 & 40,3 & 52,7 & 3,1 & 3,1 & 0,8 & 129 \\
\hline 22 & 42,3 & 47,2 & 4,0 & 6,5 & - & 123 \\
\hline 23 & 49,2 & 37,7 & 2,4 & 7,4 & 3,2 & 122 \\
\hline 24 & 37,9 & 50,0 & 2,4 & 9,7 & - & 124 \\
\hline 25 & 46,7 & 43,3 & 2,5 & 5,8 & 1,6 & 120 \\
\hline 26 & 47,5 & 45,0 & 4,1 & 3,3 & - & 120 \\
\hline 27 & 43,3 & 50,0 & 2,5 & 3,3 & 0,8 & 120 \\
\hline 28 & 55,8 & 38,3 & 1,6 & 4,2 & - & 120 \\
\hline 29 & 51,7 & 38,3 & 5,9 & 3,3 & 0,8 & 120 \\
\hline 30 & 47,5 & 42,6 & 5,8 & 2,5 & 1,6 & 122 \\
\hline 31 & 44,7 & 39,8 & 8,1 & 5,7 & 1,6 & 123 \\
\hline 32 & 44,5 & 41,4 & 11,0 & 2,3 & 0,8 & 128 \\
\hline 33 & 50,8 & 35,4 & 6,9 & 6,9 & - & 130 \\
\hline 34 & 37,1 & 49,2 & 6,8 & 5,3 & 1,6 & 132 \\
\hline 35 & 41,2 & 46,3 & 7,4 & 5,1 & - & 136 \\
\hline 36 & 42,9 & 43,6 & 9,3 & 3,6 & 0,7 & 140 \\
\hline 37 & 45,5 & 39,4 & 10,6 & 3,8 & 0,8 & 132 \\
\hline 38 & 45,1 & 44,3 & 6,6 & 4,1 & - & 122 \\
\hline 39 & 46,7 & 43,3 & 6,6 & 3,3 & - & 120 \\
\hline 40 & 48,8 & 37,2 & 10,8 & 3,3 & - & 121 \\
\hline 41 & 53,3 & 36,7 & 9,2 & 0,8 & - & 120 \\
\hline 42 & 39,2 & 40,8 & 16,7 & 3,3 & - & 120 \\
\hline
\end{tabular}

Houve maior concentração de placentas grau 0 até a $31^{\text {a }}$ semana, em contraposição à placenta grau III, que aumentou sua freqüência com o progredir da gestação, com 66,7\% às 42 semanas (Tabela 2).

Houve um aumento estatisticamente significativo da espessura da placenta com a evolução da idade gestacional, com aumentos de $1 \mathrm{~mm}$ em média por semana. Esta evolução persistiu até ao redor da 35 a semana, a partir da qual o aumento foi menor, de $0,3 \mathrm{~mm}$ por semana, até as 42 semanas (Figura 1).

A apresentação cefálica teve sua freqüência significativamente aumentada com a evolução da gestação, atingindo $99,2 \%$ na $42^{\text {a }}$ semana. A apresentação pélvica foi observada entre $31,5 \%$ das gestações de 20 semanas, somente em 0,8\% na $42^{\mathrm{a}}$ semana. A apresentação córmica mantevese com uma freqüência baixa durante todas as idades gestacionais, sem nenhum caso ao termo (Figura 2).

A posição fetal mais freqüente foi a de dorso lateral esquerdo, com $52,5 \%$, seguida da posição dorso lateral direito com $34,4 \%$. Este predomínio de posição manteve-se durante todas as idades gestacionais (Tabela 3 ). 


\begin{tabular}{|c|c|c|c|c|c|}
\hline \multirow{2}{*}{$\begin{array}{c}\text { Idade } \\
\text { Gestacional } \\
\text { (sem) }\end{array}$} & \multicolumn{4}{|c|}{ Grau placentário* } & \multirow[b]{2}{*}{$\mathrm{n}$} \\
\hline & 0 & I & II & III & \\
\hline 20 & 100,0 & - & - & - & 124 \\
\hline 21 & 100,0 & - & - & - & 129 \\
\hline 22 & 100,0 & - & - & - & 123 \\
\hline 23 & 100,0 & - & - & - & 122 \\
\hline 24 & 100,0 & - & - & - & 124 \\
\hline 25 & 94,2 & 5,8 & - & - & 120 \\
\hline 26 & 95,8 & 4,2 & - & - & 120 \\
\hline 27 & 95,8 & 4,2 & - & - & 120 \\
\hline 28 & 90,0 & 10,0 & - & - & 120 \\
\hline 29 & 75,8 & 24,2 & - & - & 120 \\
\hline 30 & 65,6 & 34,4 & - & - & 122 \\
\hline 31 & 51,2 & 48,8 & - & - & 123 \\
\hline 32 & 30,5 & 57,0 & 12,5 & - & 128 \\
\hline 33 & 26,9 & 55,4 & 17,7 & - & 130 \\
\hline 34 & 20,5 & 53,8 & 25,0 & 0,8 & 132 \\
\hline 35 & 13,2 & 44,1 & 35,3 & 7,4 & 136 \\
\hline 36 & 10,0 & 34,3 & 40,7 & 15,0 & 140 \\
\hline 37 & 2,3 & 39,4 & 38,6 & 19,7 & 132 \\
\hline 38 & 2,5 & 20,5 & 41,8 & 35,2 & 122 \\
\hline 39 & 3,3 & 19,2 & 37,5 & 40,0 & 120 \\
\hline 40 & 0,8 & 9,1 & 37,2 & 52,9 & 121 \\
\hline 41 & 1,7 & 12,5 & 30,8 & 55,0 & 120 \\
\hline 42 & 1,7 & 4,2 & 27,5 & 66,7 & 120 \\
\hline
\end{tabular}

* Classificação de Grannum, Berkowitz, Hobbins (1979)².

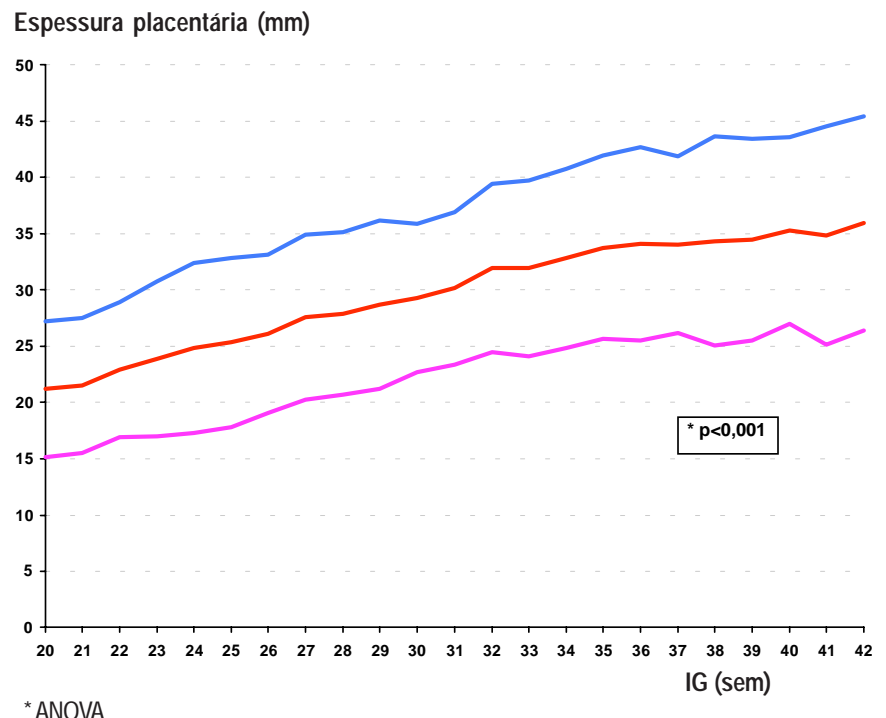

Figura 1 - Curva da espessura da placenta (média $\pm 2 D P$ ), segundo a idade gestacional, em gestantes normais.

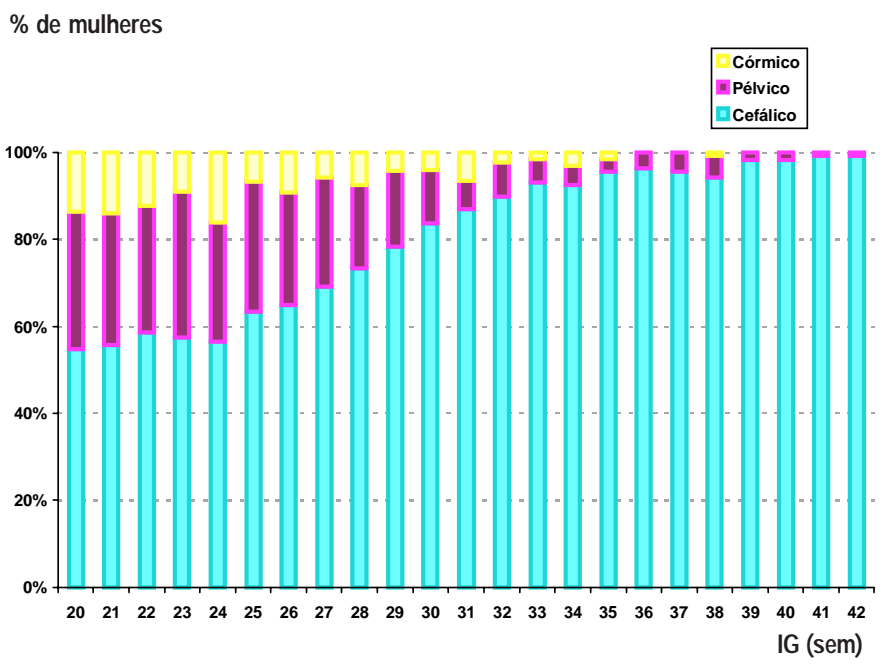

Figura 2 - Distribuição das mulheres pela frequêencia da apresentação fetal, segundo a idade gestacional.

Tabela 3 - Distribuição percentual da posição fetal, segundo a idade gestacional.

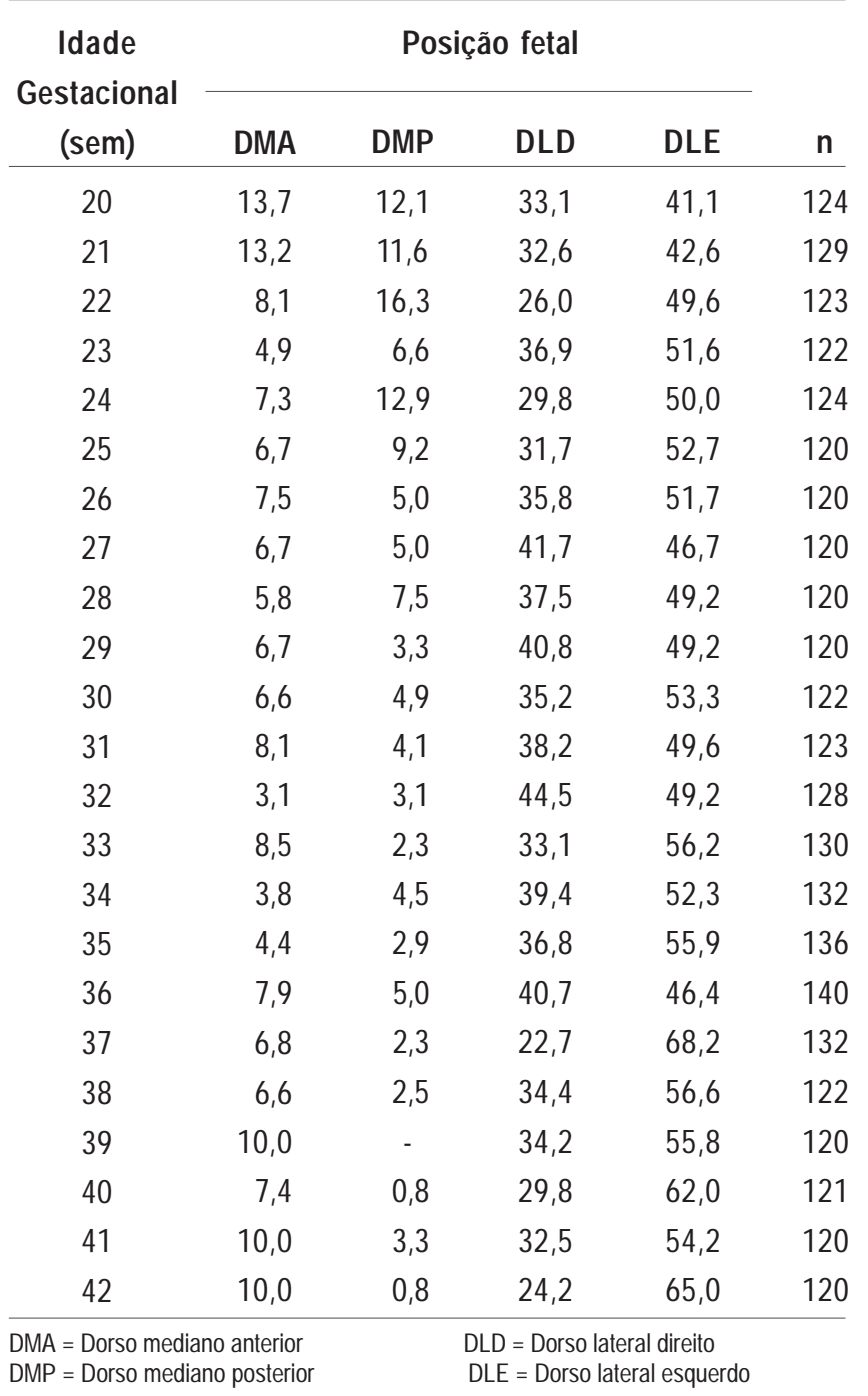




\section{Discussão}

Os resultados do presente estudo representam a tentativa de um conhecimento sistemático sobre algumas características da placenta e de estática fetal em gestantes brasileiras normais, com o objetivo de avaliar situações específicas em diferentes idades gestacionais.

Com relação à localização placentária, por exemplo, existem dúvidas se as placentas classificadas como prévias em idades gestacionais precoces persistem nesta localização até o termo. De fato, apesar do pequeno número de situações classificadas como placenta prévia, sua prevalência mostrou tendência à diminuição com a evolução da gravidez. Esta situação está de acordo com os dados disponiveis da literatura atual que indicam que o diagnóstico precoce de placenta prévia por exame ultra-sonográfico transabdominal está associado a um índice elevado de falhas na predição de placenta prévia ao termo e suas complicações, sendo que o exame realizado por via transvaginal apresentaria um melhor desempenho para este objetivo $^{6-8}$.

Os limites precisos e seguros do grau de calcificação placentária em relação à idade gestacional, considerando-se ou não o diagnóstico de senescência placentária precoce, ainda não estão totalmente estabelecidos. O diagnóstico de um envelhecimento precoce da placenta pode implicar um seguimento pré-natal com consultas mais freqüentes, na realização de outros exames para avaliação da vitalidade fetal, inclusive exames mais onerosos como a dopplervelocimetria da artéria umbilical. Além disso, é necessária uma padronização em relação ao grau placentário e idade gestacional, principalmente para serviços de hospitais-escola, para que as condutas sejam coerentes. Com padrões de normalidade estabelecidos, pode-se ainda observar como se comporta o grau de maturidade placentária em situações de hipertensão e retardo de crescimento intra-uterino, nos quais se observa freqüentemente um envelhecimento mais precoce ${ }^{2}$.

Em relação ainda ao grau placentário, os resultados deste estudo estão de acordo com os achados de Petrucha e Platt ${ }^{9}$, que descreveram que o grau placentário 0 foi mais comum até a $31^{a}$ semana, o grau I apresenta freqüência maior a partir da $32^{\mathrm{a}}$ semana e o grau II não aparecendo, usualmente, antes da $32^{\text {a }}$ semana. O grau III presente às 34 semanas não foi considerado como senescência placentária, de acordo com Grannum et al. ${ }^{2}$, tendo sido mais freqüente após a $36^{\mathrm{a}}$ semana. Apesar de estarem surgindo outras classificações paralelas em relação ao grau placentário, esta continua sendo a utilizada neste serviço e a mais difundida em todo o mundo.

A relação entre o grau placentário e a maturidade pulmonar fetal não foi objetivo deste estudo, no entanto observou-se uma maior porcentagem de placentas grau III ao termo, refletindo assim um sinal de amadurecimento fetal. O que deve ser enfatizado, entretanto, é a possibilidade de se encontrar, ainda que em menor freqüência, graus 0 e I próximo do termo e no termo, e graus II e III das 32 às 37 semanas, sem que isso represente necessariamente uma situação patológica.

No tocante à espessura placentária, verificamos uma concordância com os estudos de Hoddick et al. ${ }^{5}$, em que a espessura máxima não excede $30 \mathrm{~mm}$ às 20 semanas, e $40 \mathrm{~mm}$ antes das 40 semanas. Verificou-se também uma estreita relação entre a espessura placentária e a idade gestacional, concordando com os estudos anteriores, numa relação quase linear de crescimento de espessura da placenta com a idade gestacional. Isto facilita o diagnóstico de alterações da espessura placentária induzidas por processos patológicos, embora a variabilidade da medida deva ser considerada dentro da faixa normal, delimitada pelas linhas superior e inferior da curva (Figura 1). Além disso, a maior atenção requerida para a medida da espessura placentária da maneira descrita, evitará o diagnóstico incorreto de contrações uterinas focais e a inclusão do tecido miometrial na espessura placentária.

Em relação à apresentação fetal ao termo, observamos maior freqüência da apresentação cefálica, sendo que a apresentação pélvica ocorreu em apenas 1\%, achados estes discordantes com a literatura 4 . Embora o estudo não tenha explorado este aspecto, a única explicação plausível para um queda tão acentuada da freqüência de apresentação pélvica de 4,9\% às 38 semanas, para ao redor de 1\% às 40-41 semanas, é a provável interrupção destas gestações por meio de cesárea, procedimento quase rotineiramente utilizado nesta apresentação em nosso meio, à exceção de alguns poucos serviços universitários que ainda permitem a via vaginal para o parto pélvico.

Constatamos, assim, que os fatores estudados, características ecográficas da placenta, posição e apresentação fetal, tiveram distribuição similar à esperada para populações normais, apesar das diferenças étnicas e demográficas entre as populações já estudadas anteriormente e a atual. Espera-se que a demonstração de tais características possa ser de utilidade para o clínico e o médico que realiza exames ultra-sonográficos, sobretudo no sentido de não superestimar achados ecográficos que podem ser compatíveis com a 
normalidade para a idade gestacional em questão, evitando desta forma preocupações e propedêuticas desnecessárias e onerosas e, possivelmente, também algumas interrupções iatrogênicas da gestação.

\section{SUMMARY}

Purpose: to evaluate the ultrasound characteristics of normal pregnancies, according to the placental maturity, local of insertion and thickness, fetal presentation and position during the second half of pregnancy.

Methods: a descriptive study was perfomed, including at least 120 measures in each gestational age, in 2,868 normal pregnant women from Campinas, Brazil, studied through routine obstetric ultrasound examinations, with fetal biometry and placental evaluation, applying Grannum, Berkowitz, Hobbins (1979) criteria for placental maturity. Placental thickness was measured at the cord insertion site.

Results: grade 0 placentas were more frequent up to 31 weeks and grade I after 32 weeks. Grade II did not appear until 32 weeks and grade III was more frequent after 36 weeks. The placental thickness significantly increased with gestational age and the most frequent placental locations were anterior and posterior. The cephalic presentation was the most frequent all gestational ages, with only $1 \%$ of breech presentation at term. The most frequent fetal position was fetal spine left side, followed by right side.

Conclusions: the studied factors showed a similar distribution to that expected for normal populations and could be used as a standard for the Brazilian population.

KEY WORDS: Placenta. Normal pregnancy. Ultrasound.

\section{Referências}

1. Myles TD, Strassner HT. Four-quadrant assessment of amniotic fluid volume: distribution's role in predicting fetal outcome. Obstet Gynecol 1992; 80: 769-74.

2. Grannum PAT, Berkowitz RL, Hobbins JC. The ultrasonic changes in the maturing placenta and their relationship to fetal pulmonic maturity. Am J Obstet Gynecol 1979; 133: 915-22.

3. Kuhlmann RS, Warsof S. Ultrasound of the placenta. Clin Obstet Gynecol 1996; 39: 519-34.

4. Cunningham FG, MacDonald PC, Gant NF, Leveno KJ, Gilstrap III LC, Hankins GDV, Clark SL. Obstetrical hemorrhage. In: Willians Obstetrics. $20^{\text {th }}$ Edition. Stamford: Appleton \& Lange; 1997. p.755-60.

5. Hoddick WK, Mahoony BS, Callen PW, Filly RA. Placental thickness. J Ultrasound Med 1985; 4: 479-82.

6. Zelop CC, Bromley B, Frigoletto FD, Benacerraf BR. Second trimester sonographically diagnosed placenta previa: prediction of persistent previa at birth. Int J Gynaecol Obstet 1994; 44: 207-10.

7. Lauria MR, Smith RS, Treadwell MC, Comstock CH, Kirk JS, Lee W, Bottoms SF. The use of secondtrimester transvaginal sonography to predict placenta previa. Ultrasound Obstet Gynecol 1996; 8: 337-40.

8. Taipale P, Hiilesmaa V, Ylostalo P. Transvaginal ultrasonography at $18-23$ weeks in predicting placenta previa at delivery. Ultrasound Obstet Gynecol 1998; 12: 422-5.

9. Petrucha RA, Platt LD. Relationship of placental grade to gestational age. Am J Obstet Gynecol 1982; 144: 733-5.

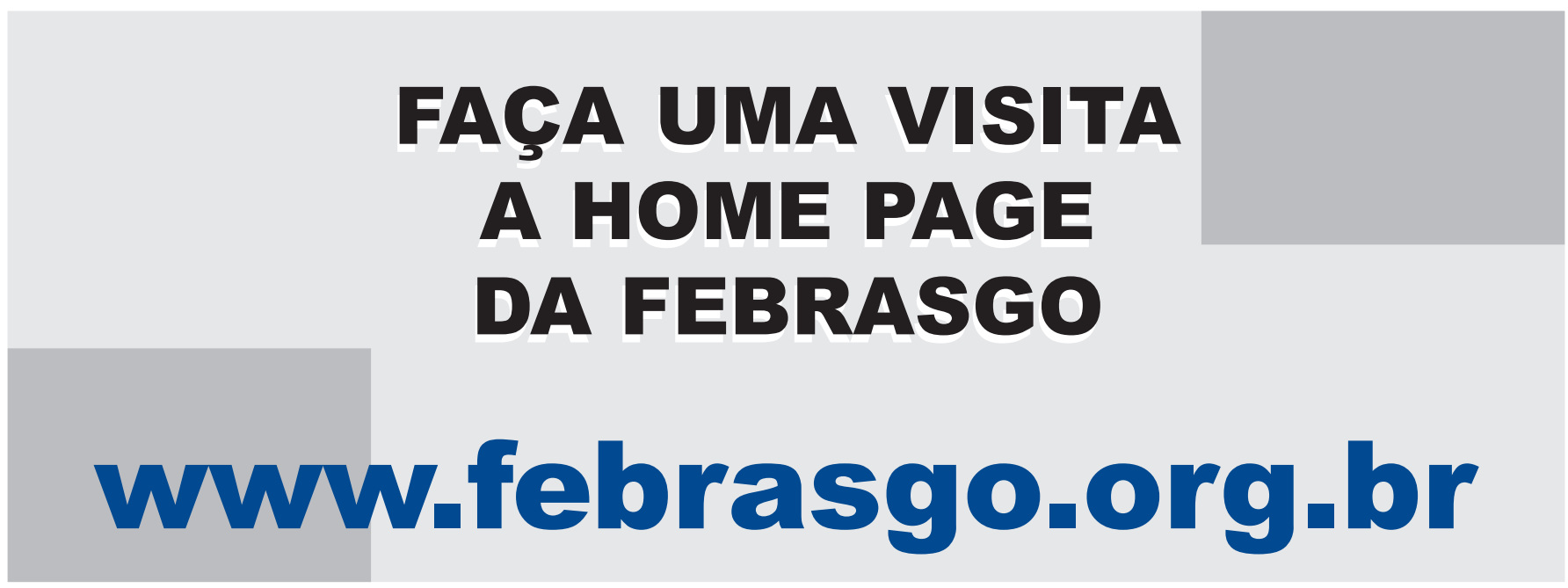

